\title{
Generalized Measurement and Conclusive Teleportation with Nonmaximal Entanglement
}

\author{
Hyunjae Kim, Yong Wook Cheong, and Hai-Woong Lee \\ Department of Physics, Korea Advanced Institute of Science and Technology, Daejeon 305-701, Korea
}

(Dated: August 15, 2018)

\begin{abstract}
We present linear optical schemes to perform generalized measurements for conclusive teleportation when the sender and the receiver share nonmaximal entanglement resulting from amplitude errors during propagation or generation. Three different cases are considered for which the states to be teleported are unknown superpositions of (a) single-photon and vacuum states, (b) verticallypolarized and horizontally-polarized photon states, and (c) two coherent states of opposite phases. The generalized measurement scheme for each case is analyzed, which indicates that the success probability is much more resistant to amplitude errors for case (c) than for case (a) or (b).
\end{abstract}

\section{INTRODUCTION}

Since its first proposal in 1993[1], quantum teleportation has been the subject of intensive study, both theoretical and experimental. Up to now, quantum teleportation of superposed photon polarization states [2], superposed one-photon and vacuum states [3], and continuous variable light states 4] has been demonstrated experimentally. Although teleportation can be achieved ideally with the success probability of 1 and the fidelity of 1 , there exist many practical limitations, such as various sources of decoherence, finite efficiency of detectors, lack of reliable single-photon sources, difficulty with perfect Bell-state measurements, and nonmaximal entanglement, that need to be overcome before the experimental performance can match ideal theoretical predictions.

The process of teleportation may be regarded as the transfer of quantum information through a quantum channel. When the quantum channel is provided by a maximally entangled pair, quantum teleportation faithfully transmits the quantum information. Amplitude errors during generation or distribution of entanglement, however, degrade the degree of entanglement. The practical question of importance is thus what one should or can do when the sender, Alice, and the receiver, Bob, share nonmaximal entanglement. Different strategies should be adopted by Alice and Bob depending upon the situation. They may choose the teleportation scheme that would yield the maximum average fidelity. In some cases, however, what is required is not to obtain the maximum information but to avoid making any mistake even at the expense of lowered success probability. In such cases, they can adopt the strategy of "conclusive teleportation" [5], in which the teleportation succeeds with the probability less than one but Alice knows when it succeeds or not, and when it succeeds the fidelity of the teleported state is one. There are three approaches to conclusive teleportation that Alice and Bob can take. First, they may try to extract maximally entangled pairs out of the given nonmaximally entangled pairs through the process of entanglement concentration [6], and perform teleportation with the concentrated pairs. The number of the concentrated pairs is less than the number of the original nonmaximally entangled pairs, i.e., the probability for concentration is less than one, but the fidelity of the teleported state using the concentrated pair as the quantum channel is one. Second, Alice may perform the standard Bell-state measurements directly with the nonmaximally entangled pairs and let Bob apply an appropriate unitary transformation with the aid of an auxiliary qubit to obtain the desired state probabilistically 7 . The success probability is less than one, but Bob knows whether he obtained the desired state or not by observing the state of the auxiliary qubit. Third, Alice may perform "generalized measurements" 8] (or positive operator valued measurement) upon the nonmaximally entangled pairs that distinguish nonorthogonal "Bell-type" states conclusively with a certain probability less than one 5]. When the measurement outcome corresponds to the conclusive event, the teleportation succeeds with the fidelity of 1 .

It is this third approach we wish to study in detail here. We construct linear optical schemes that enable one to perform the desired generalized measurements for three different cases for which two basis states for a qubit are given by (a) $|1\rangle$, a single-photon state, and $|0\rangle$, the vacuum state, (b) $|V\rangle$, a vertically-polarized photon state, and $|H\rangle$, a horizontally-polarized photon state, and (c) $|\alpha\rangle$ and $|-\alpha\rangle$, two coherent light states with opposite phases. We analyze and compare the generalized measurement process for conclusive teleportation for the three cases. The reason we study and compare these three cases is that, although the basic principles governing the generalized measurement and teleportation processes may be the same regardless of the choice of qubit states, one may have significant advantages over another when the actual experimental implementation of the scheme is considered. We need only to recall that, in quantum key distribution, the choice of $|1\rangle$ and $|0\rangle$ has led to the phase coding scheme which offers significant practical advantages over the polarization coding scheme resulting from the choice of $|V\rangle$ and $|H\rangle$. In case of quantum teleportation with maximal entanglement, investigations in the past have revealed that the three cases require different levels of experimental 
sophistication in Bell-state measurements and in transformations 1, 2, 3, 9, 10, 11, 12]. For exa polarizing beam splitters needed in Bell-state mec ments for case (b) are not required for case (a); in detectors in case (a) need to distinguish a single ton from two $[9]$. The state transformations requir cases (a) and (b) are all unitary, but some transf tions involved in case (c) are nonunitary 11, 12]. makes case (c) still particularly interesting is the that four "quasi Bell states" can in principle be all d guished with a high probability using only linear o means 11, 12], whereas for cases (a) and (b) only t the four Bell states can be distinguished linear opt: Since in general a different choice of qubit states lec a different experimental setup for generalized mec ments with different optical devices which may res differently to an attempt to compensate for amp. errors that caused nonmaximal entanglement, it $\mathrm{s}$ be of interest to analyze and compare in detail the $\epsilon$ imental schemes for conclusive teleportation for dif choices of qubit states. Such comparison and an have indeed led to the main result of our work the success probability is much more resistant to amp. errors for case (c) than for case (a) or (b).

\section{GENERALIZED MEASUREMENT AN CONCLUSIVE TELEPORTATION}

In this section we construct linear optical schem generalized measurements and analyze the gener: measurements that need to be performed with the posed schemes, for the three different cases (a), (b (c) mentioned in the previous section.

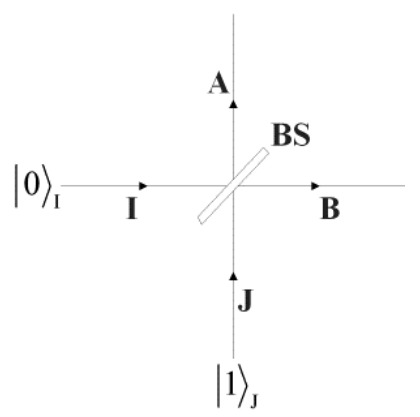

FIG. 1: Generation of the nonmaximally entangled state $\left(\cos \eta|1\rangle_{A}|0\rangle_{B}-\sin \eta|0\rangle_{A}|1\rangle_{B}\right)$. BS represents a beam splitter of transmission coefficient $t=\cos \eta$ and reflection coefficient $r=\sin \eta$, with input ports $\mathbf{I}$ and $\mathbf{J}$ and output ports $\mathbf{A}$ and $\mathbf{B}$. A single photon is incident on BS through the input port $\mathbf{J}$.

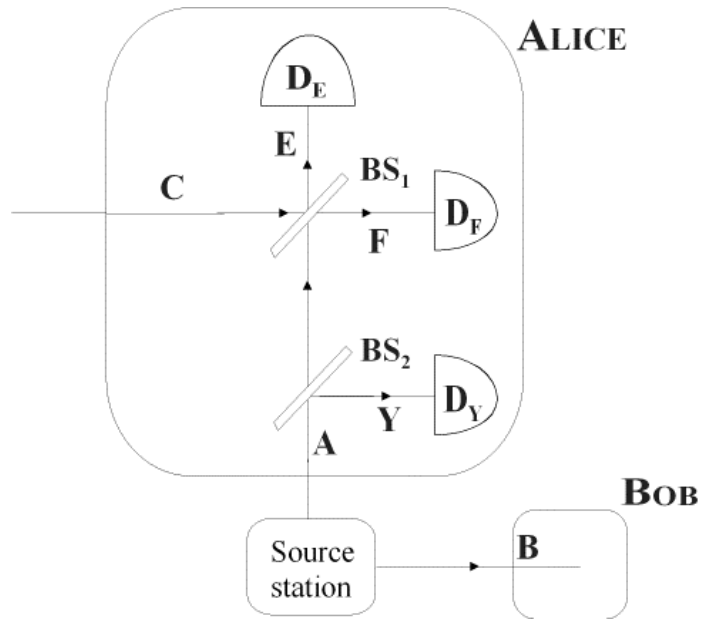

FIG. 2: Linear optical scheme for a conclusive teleportation of the state $\left(x|1\rangle_{C}+y|0\rangle_{C}\right)$. The source station generates a nonmaximally entangled state $\left|\Psi_{\eta}^{-}\right\rangle_{A B}=\cos \eta|1\rangle_{A}|0\rangle_{B}-$ $\sin \eta|0\rangle_{A}|1\rangle_{B}$. $\mathbf{D}_{\mathbf{E}}, \mathbf{D}_{\mathbf{F}}$ and $\mathbf{D}_{\mathbf{Y}}$ are detectors, $\mathbf{B S}_{\mathbf{1}}$ is a $50 / 50$ beam splitter, and $\mathbf{B S}_{\mathbf{2}}$ is a beam splitter of transmission coefficient $t=\tan \eta$.

\section{A. Conclusive teleportation of the state $(x|1\rangle+y|0\rangle)$}

We first consider the case when two basis states for a qubit are the single-photon and vacuum states, $|1\rangle$ and $|0\rangle$. Let us suppose that Alice and Bob are given a nonmaximally entangled state

$$
\left|\Psi_{\eta}^{-}(1,0)\right\rangle_{A B} \equiv \cos \eta|1\rangle_{A}|0\rangle_{B}-\sin \eta|0\rangle_{A}|1\rangle_{B},
$$

where the subscripts $\mathbf{A}$ and $\mathbf{B}$ signify that the beams $\mathbf{A}$ and $\mathbf{B}$ that contain the entangled state belong to Alice and Bob, respectively. The parameter $\eta$ determines the degree of entanglement. Without loss of generality, we assume that $\cos \eta>\sin \eta\left(0<\eta<\frac{\pi}{4}\right)$. The entangled state of Eq.(11) results when a photon is passed through a beam splitter whose transmission and reflection coefficients deviate from $\frac{1}{\sqrt{2}}$. As shown in Fig [ when a single photon is incident on a beam splitter of transmission coefficient $t=\cos \eta$ and reflection coefficient $r=\sqrt{1-t^{2}}=\sin \eta$, the output state is given by Eq.(1).

Alice has another beam $\mathbf{C}$ in an unknown state $\left(x|1\rangle_{C}+y|0\rangle_{C}\right) \equiv\left(\begin{array}{l}x \\ y\end{array}\right)_{C}$ with unknown coefficients $x$ and $y$, which she wishes to teleport to Bob. The total state of the beams A, B and $\mathbf{C},|\Psi\rangle_{A B C}=\left|\Psi_{\eta}^{-}(1,0)\right\rangle_{A B}\left(\begin{array}{l}x \\ y\end{array}\right)_{C}$, can be expanded as (we adopt bracket notations for two-particle states and vector notations for one-particle states)

$$
\begin{aligned}
|\Psi\rangle_{A B C}= & \frac{1}{2}\left[\left|\Psi_{\eta}^{-}\right\rangle_{A C}\left(\begin{array}{c}
x \\
y
\end{array}\right)_{B}-\left|\Psi_{\eta}^{+}\right\rangle_{A C}\left(\begin{array}{c}
x \\
-y
\end{array}\right)_{B}\right. \\
& \left.+\left|\Phi_{\eta}^{-}\right\rangle_{A C}\left(\begin{array}{c}
y \\
x
\end{array}\right)_{B}+\left|\Phi_{\eta}^{+}\right\rangle_{A C}\left(\begin{array}{c}
-y \\
x
\end{array}\right)_{B}\right],
\end{aligned}
$$


where the four "Bell-type states" are defined by

$$
\begin{aligned}
\left|\Psi_{\eta}^{\mp}\right\rangle_{A C} & \equiv\left|\Psi_{\eta}^{\mp}(1,0)\right\rangle_{A C} \\
& =\cos \eta|1\rangle_{A}|0\rangle_{C} \mp \sin \eta|0\rangle_{A}|1\rangle_{C}, \\
\left|\Phi_{\eta}^{\mp}\right\rangle_{A C} & \equiv\left|\Phi_{\eta}^{\mp}(1,0)\right\rangle_{A C} \\
& =\cos \eta|1\rangle_{A}|1\rangle_{C} \mp \sin \eta|0\rangle_{A}|0\rangle_{C} .
\end{aligned}
$$

When $\eta=\frac{\pi}{4}$, these four states reduce to the standard Bell states $\left|\Psi^{\mp}\right\rangle_{A C}=\frac{1}{\sqrt{2}}\left(|1\rangle_{A}|0\rangle_{C} \mp|0\rangle_{A}|1\rangle_{C}\right)$ and $\left|\Phi^{\mp}\right\rangle_{A C}=\frac{1}{\sqrt{2}}\left(|1\rangle_{A}|1\rangle_{C} \mp|0\rangle_{A}|0\rangle_{C}\right)$.

The conclusive teleportation protocol calls for a generalized measurement which unambiguously distinguishes at least two of the four nonorthogonal states of Eq.(3) at the price of occasional failures 5. We recall that the linear optical scheme that distinguishes two of the standard Bell states $\left|\Psi^{\mp}\right\rangle$ and $\left|\Phi^{\mp}\right\rangle$ consists of a $50 / 50$ beam splitter and two detectors $\left(\mathbf{B S}_{\mathbf{1}}, \mathbf{D}_{\mathbf{E}}\right.$ and $\mathbf{D}_{\mathbf{F}}$ of Fig 2) 9]. The generalized measurement to distinguish unambiguously two of the four states of Eq.(3) can be accomplished by adding to them another beam splitter of transmission coefficient $t=\tan \eta$ and a third detector $\left(\mathbf{B S}_{\mathbf{2}}\right.$ and $\mathbf{D}_{\mathbf{Y}}$ of Fig 2 2$)$ in the path of the beam $\mathbf{A}$, as shown in Fig 2 By utilizing the relations between photon creation operators of different modes given by $A^{\dagger}=\frac{1}{\sqrt{2}}\left(t E^{\dagger}-t F^{\dagger}\right)-r Y^{\dagger}, C^{\dagger}=\frac{1}{\sqrt{2}}\left(E^{\dagger}+F^{\dagger}\right)$, where $r$ is the reflection coefficient $r=\sqrt{1-\tan ^{2} \eta}$, we obtain that the state $|\Psi\rangle_{A B C}=\left|\Psi_{\eta}^{-}\right\rangle_{A B}\left(\begin{array}{l}x \\ y\end{array}\right)_{C}$ is transformed, via the action of the beam splitters $\mathbf{B S}_{\mathbf{1}}$ and $\mathbf{B S}_{\mathbf{2}}$, into $|\Psi\rangle_{E F Y B}$, where

$$
\begin{aligned}
|\Psi\rangle_{E F Y B}= & \frac{1}{2}\left\{\left(-\sqrt{2} \sin \eta|0\rangle_{E}|1\rangle_{F}|0\rangle_{Y}-\sqrt{\cos 2 \eta}|0\rangle_{E}|0\rangle_{F}|1\rangle_{Y}\right)\left(\begin{array}{c}
x \\
y
\end{array}\right)_{B}\right. \\
& -\left(\sqrt{2} \sin \eta|1\rangle_{E}|0\rangle_{F}|0\rangle_{Y}-\sqrt{\cos 2 \eta}|0\rangle_{E}|0\rangle_{F}|1\rangle_{Y}\right)\left(\begin{array}{c}
x \\
-y
\end{array}\right)_{B} \\
& +\left(\sin \eta\left|\Psi^{-}(2,0)\right\rangle_{E F}|0\rangle_{Y}-\sqrt{\cos 2 \eta}\left|\Psi^{+}(1,0)\right\rangle_{E F}|1\rangle_{Y}-\sin \eta|0\rangle_{E}|0\rangle_{F}|0\rangle_{Y}\right)\left(\begin{array}{c}
y \\
x
\end{array}\right) \\
& \left.+\left(\sin \eta\left|\Psi^{-}(2,0)\right\rangle_{E F}|0\rangle_{Y}-\sqrt{\cos 2 \eta}\left|\Psi^{+}(1,0)\right\rangle_{E F}|1\rangle_{Y}+\sin \eta|0\rangle_{E}|0\rangle_{F}|0\rangle_{Y}\right)\left(\begin{array}{c}
-y \\
x
\end{array}\right)_{B}\right\},
\end{aligned}
$$

where the state $\left|\Psi^{-}(2,0)\right\rangle_{E F}$ denotes the state $\frac{1}{\sqrt{2}}\left(|2\rangle_{E}|0\rangle_{F}-|0\rangle_{E}|2\rangle_{F}\right)$.

The generalized measurement consists of measuring the number of photons that arrive at each of the three detectors $\mathbf{D}_{\mathbf{E}}, \mathbf{D}_{\mathbf{F}}$ and $\mathbf{D}_{\mathbf{Y}}$. It is clear from Eq. (44) that the teleportation succeeds either if $\mathbf{D}_{\mathbf{F}}$ registers one photon and $\mathbf{D}_{\mathbf{E}}$ and $\mathbf{D}_{\mathbf{Y}}$ none or if $\mathbf{D}_{\mathbf{E}}$ registers one photon and $\mathbf{D}_{\mathbf{F}}$ and $\mathbf{D}_{\mathbf{Y}}$ none. All other measurement results correspond to inconclusive events. It is also clear from Eq. (4) that the success probability for the conclusive teleportation is $P=\sin ^{2} \eta$. When $\eta=\frac{\pi}{4}$, i.e., when Alice and Bob share maximal entanglement, $P=\frac{1}{2}$ consistent with the previous analysis 9 . As the degree of entanglement decreases, the success probability decreases, too. Nevertheless, the fidelity $F$ remains 1 . We note that a successful conclusive teleportation for the present case requires detectors $\mathbf{D}_{\mathbf{E}}$ and $\mathbf{D}_{\mathbf{F}}$ to discriminate between 0,1 and 2 photons and detector $\mathbf{D}_{\mathbf{Y}}$ to discriminate between 0 and 1 photon.

\section{B. Conclusive teleportation of the state} $(x|V\rangle+y|H\rangle)$

We next consider the case when two basis states for a qubit are the vertically-polarized and horizontally- polarized photon states $|V\rangle$ and $|H\rangle$. The nonmaximally entangled state $\left|\Psi_{\eta}^{-}(V, H)\right\rangle_{A B}$ for two photons $\mathbf{A}$ and $\mathbf{B}$ now takes the form of Eq.(11) with $|1\rangle$ and $|0\rangle$ replaced by $|V\rangle$ and $|H\rangle$. This type of nonmaximally entangled state can be produced by using a spontaneous parametric down-conversion source which consists of two crystals with their optic axis oriented orthogonal to each other, as demonstrated by White et al [13]. The unknown polarization state of the photon that Alice wishes to teleport to Bob is $\left(x|V\rangle_{C}+y|H\rangle_{C}\right)$, which we again denote by $\left(\begin{array}{l}x \\ y\end{array}\right)_{C}$. The four Bell-type states that need to be distinguished are now

$$
\begin{aligned}
\left|\Psi_{\eta}^{\mp}\right\rangle_{A C} & \equiv\left|\Psi_{\eta}^{\mp}(V, H)\right\rangle_{A C} \\
& =\cos \eta|V\rangle_{A}|H\rangle_{C} \mp \sin \eta|H\rangle_{A}|V\rangle_{C}, \\
\left|\Phi_{\eta}^{\mp}\right\rangle_{A C} & \equiv\left|\Phi_{\eta}^{\mp}(V, H)\right\rangle_{A C} \\
& =\cos \eta|V\rangle_{A}|V\rangle_{C} \mp \sin \eta|H\rangle_{A}|H\rangle_{C} .
\end{aligned}
$$

We recall that the linear optical scheme that distinguishes two of the standard Bell states $\left|\Psi^{\mp}\right\rangle=\frac{1}{\sqrt{2}}(|V\rangle|H\rangle \mp|H\rangle|V\rangle)$ and $\left|\Phi^{\mp}\right\rangle=$ $\frac{1}{\sqrt{2}}(|V\rangle|V\rangle \mp|H\rangle|H\rangle)$ consists of a $50 / 50$ beam splitter, two polarizing beam splitters and four detectors $\left(\mathbf{B S}_{1}, \mathbf{P B S}_{1}, \mathbf{P B S}_{2}, \mathbf{D}_{\mathrm{E} 1}, \mathbf{D}_{\mathrm{E} 2}, \mathbf{D}_{\mathrm{F} 1}\right.$ and $\mathbf{D}_{\mathrm{F} 2}$ of Fig 30 10. The corresponding generalized measurement that distinguishes two of the states of Eq.(5) can 
be accomplished by adding another beam splitter of transmission coefficient $t=\tan \eta$ and two additional polarizing beam splitters $\left(\mathbf{B S}_{\mathbf{2}}, \mathbf{P B S}_{\mathbf{3}}\right.$ and $\mathbf{P} \mathbf{B S}_{\mathbf{4}}$ of Fig [3] in the path of photon $\mathbf{A}$, as shown in Fig [3] The polarizing beam splitters transmit vertically-polarized photons and reflect horizontally-polarized photons. The combination of $\mathbf{P B S} \mathbf{S}_{\mathbf{3}}$ and $\mathbf{B S}_{\mathbf{2}}$ thus acts to reduce the amplitude of the vertically-polarized component of wave
A by a factor of $t=\tan \eta$, while leaving the amplitude of the horizontally-polarized component unaltered. In fact, one obtains through a straightforward calculation that the state $|\Psi\rangle_{A B C}=\left|\Psi_{\eta}^{-}(V, H)\right\rangle_{A B}\left(\begin{array}{l}x \\ y\end{array}\right)_{C}$ is transformed, via the action of $\mathbf{P} \mathbf{B S}_{\mathbf{3}}, \mathbf{B S}_{\mathbf{2}}, \mathbf{P} \mathbf{B} \mathbf{S}_{\mathbf{4}}$ and $\mathbf{B} \mathbf{S}_{\mathbf{1}}$, into the state $|\Psi\rangle_{E F Y B}$, where

$$
\begin{aligned}
|\Psi\rangle_{E F Y B}= & \frac{1}{2}\left\{\left[\sqrt{2} \sin \eta\left|\Psi^{-}(V, H)\right\rangle_{E F}|0\rangle_{Y}+\sqrt{\cos 2 \eta}\left|\Psi^{+}(H, 0)\right\rangle_{E F}|V\rangle_{Y}\right]\left(\begin{array}{c}
x \\
y
\end{array}\right)_{B}\right. \\
& -\left[\sqrt{2} \sin \eta\left|\Psi^{-}(V H, 0)\right\rangle_{E F}|0\rangle_{Y}+\sqrt{\cos 2 \eta}\left|\Psi^{+}(H, 0)\right\rangle_{E F}|V\rangle_{Y}\right]\left(\begin{array}{c}
x \\
-y
\end{array}\right)_{B} \\
& +\left[\sin \eta\left(\left|\Psi^{-}(2 V, 0)\right\rangle_{E F}-\left|\Psi^{-}(2 H, 0)\right\rangle_{E F}\right)|0\rangle_{Y}+\sqrt{\cos 2 \eta}\left|\Psi^{+}(V, 0)\right\rangle_{E F}|V\rangle_{Y}\right]\left(\begin{array}{c}
y \\
x
\end{array}\right)_{B} \\
& \left.+\left[\sin \eta\left(\left|\Psi^{-}(2 V, 0)\right\rangle_{E F}+\left|\Psi^{-}(2 H, 0)\right\rangle_{E F}\right)|0\rangle_{Y}+\sqrt{\cos 2 \eta}\left|\Psi^{+}(V, 0)\right\rangle_{E F}|V\rangle_{Y}\right]\left(\begin{array}{c}
-y \\
x
\end{array}\right)_{B}\right\},
\end{aligned}
$$

The Bell-state notations used in Eq. (6) should be self-evident. For example, $\left|\Psi^{-}(V H, 0)\right\rangle_{E F}=$ $\frac{1}{\sqrt{2}}\left(|V H\rangle_{E}|0\rangle_{F}-|0\rangle_{E}|V H\rangle_{F}\right)$, where $|0\rangle_{E}$ means that there is neither vertically-polarized nor horizontallypolarized photon of mode $\mathbf{E}$, and $|V H\rangle_{E}$ stands for the state of one vertically-polarized photon and one horizontally-polarized photon of mode E. Similarly, $\left|\Psi^{-}(2 V, 0)\right\rangle_{E F}=\frac{1}{\sqrt{2}}\left(|2 V\rangle_{E}|0\rangle_{F}-|0\rangle_{E}|2 V\rangle_{F}\right)$, where $|2 V\rangle_{E}$ stands for the state of two vertically-polarized photons of mode E. It is clear from Eq.(6) that the teleportation succeeds if detectors $\mathbf{D}_{\mathbf{E} 1}$ and $\mathbf{D}_{\mathbf{F} 2}$ register a photon each or if detectors $\mathbf{D}_{\mathbf{E} 2}$ and $\mathbf{D}_{\mathbf{F} 1}$ register a photon each (corresponding to the term $\left|\Psi^{-}(V, H)\right\rangle_{E F}$ in the square bracket of the first line of Eq.(6) ), or if detectors $\mathbf{D}_{\mathbf{E} 1}$ and $\mathbf{D}_{\mathbf{E} 2}$ register a photon each, or if detectors $\mathbf{D}_{\mathbf{F} 1}$ and $\mathbf{D}_{\mathbf{F} 2}$ register a photon each (corresponding to the term $\left|\Psi^{-}(V H, 0)\right\rangle_{E F}$ in the square bracket of the second line of Eq.(6)). All other measurement results correspond to inconclusive events. It is also clear from Eq.(6) that, as in the previous case where the basis states for a qubit are the single-photon and vacuum states, the success probability is $\sin ^{2} \eta$ and the fidelity is 1 . The present case, however, holds an interesting advantage over the previous case in that there is no need for a detector to detect photons of mode $\mathbf{Y}$. The combination $|V\rangle_{E}|H\rangle_{F}$, $|H\rangle_{E}|V\rangle_{F},|V\rangle_{E}|H\rangle_{E}$, or $|V\rangle_{F}|H\rangle_{F}$ does not appear in the third or fourth line of Eq.(6). Another important advantage is that, in the present case, detectors do not need to distinguish between one and two photons. For example, a click each at detectors $\mathbf{D}_{\mathbf{E} 1}$ and $\mathbf{D}_{\mathbf{F} 2}$ along with no detection of photons at detectors $\mathbf{D}_{\mathbf{E} 2}$ and $\mathbf{D}_{\mathbf{F} 1}$ indicates conclusively that the state of photon $\mathbf{B}$ is $\left(\begin{array}{l}x \\ y\end{array}\right)_{B}=x|V\rangle_{B}+y|H\rangle_{B}$.

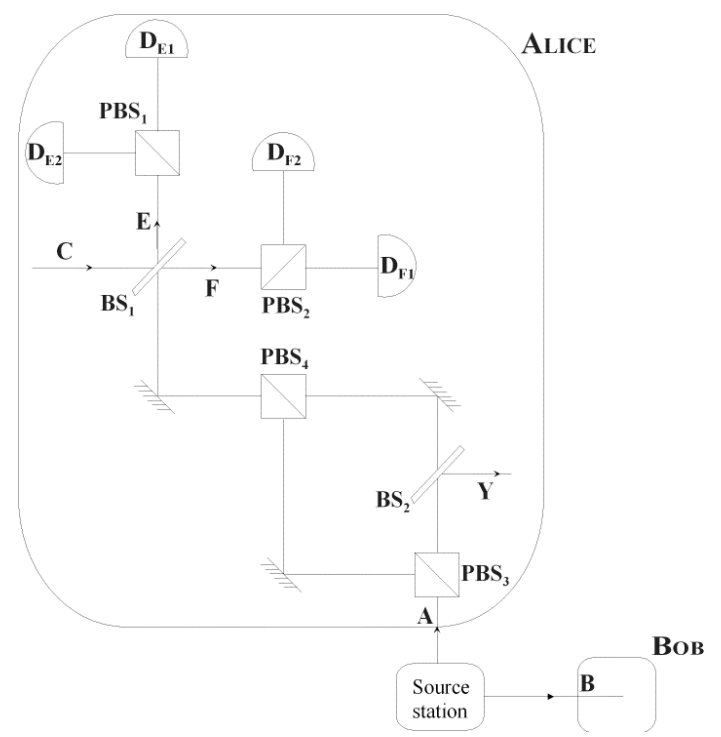

FIG. 3: Linear optical scheme for a conclusive teleportation of the state $\left(x|V\rangle_{C}+y|H\rangle_{C}\right)$. The source station generates a nonmaximally entangled state $\left|\Psi_{\eta}^{-}\right\rangle_{A B}=\cos \eta|V\rangle_{A}|H\rangle_{B}-$ $\sin \eta|H\rangle_{A}|V\rangle_{B}$. $\mathbf{D}_{\mathbf{E} 1}, \mathbf{D}_{\mathbf{E} 2}, \mathbf{D}_{\mathbf{F} 1}$, and $\mathbf{D}_{\mathbf{F} 2}$ are detectors, $\mathbf{P B S}_{1}, \mathbf{P B S}_{\mathbf{2}}, \mathbf{P B S _ { 3 }}$ and $\mathbf{P B S} \mathbf{B}_{4}$ are polarizing beam splitters, $\mathbf{B S}_{\mathbf{1}}$ is a $50 / 50$ beam splitter, and $\mathbf{B} \mathbf{S}_{\mathbf{2}}$ is a beam splitter of transmission coefficient $t=\tan \eta$.

\section{Conclusive teleportation of the state $(x|\alpha\rangle+y|-\alpha\rangle)$}

Finally, we consider the case when two basis states for a qubit are the coherent states of opposite phases, $|\alpha\rangle$ and $|-\alpha\rangle$. The two basis states in this case are not orthogonal, $\langle\alpha \mid-\alpha\rangle=e^{-2|\alpha|^{2}}$; but, if $|\alpha|$ is not too small 


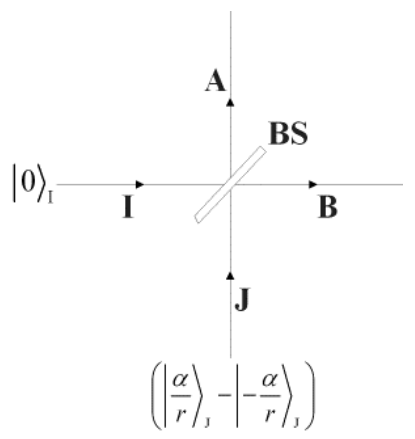

FIG. 4: Generation of the nonmaximally entangled state $N_{\alpha \beta}^{-}\left(|\alpha \tan \eta\rangle_{A}|-\alpha\rangle_{B}-|-\alpha \tan \eta\rangle_{A}|\alpha\rangle_{B}\right)$. BS represents a beam splitter of transmission coefficient $t=\sin \eta$ and reflection coefficient $r=\cos \eta$, with input ports $\mathbf{I}$ and $\mathbf{J}$ and output ports $\mathbf{A}$ and $\mathbf{B}$. The input port $\mathbf{J}$ is illuminated with light in the state $\left(\left|\frac{\alpha}{\cos \eta}\right\rangle_{J}-\left|-\frac{\alpha}{\cos \eta}\right\rangle_{J}\right)$.

$(|\alpha| \gtrsim 3)$, the overlap is sufficiently small that they can be treated orthogonal without much error. It is important to note that an amplitude loss experienced by light in a coherent state $|\alpha\rangle$ reduces not the probability amplitude associated with the state but the parameter $\alpha$ itself [14]. The nonmaximally entangled state we consider here can thus be written as

$$
\left|\Psi_{\eta}^{-}\right\rangle_{A B}=N_{\alpha \beta}^{-}\left(|\beta\rangle_{A}|-\alpha\rangle_{B}-|-\beta\rangle_{A}|\alpha\rangle_{B}\right),
$$

where $N_{\alpha \beta}^{-}$is the normalization constant $\left(N_{\alpha \beta}^{-}=1 / \sqrt{2\left[1-e^{-2\left(|\alpha|^{2}+|\beta|^{2}\right)}\right]}\right) . \quad$ We assume without loss of generality that $\alpha$ and $\beta$ are real and that $\frac{\beta}{\alpha} \equiv \tan \eta<1$. The nonmaximally entangled state of Eq. (7) can be generated by illuminating a beam splitter of transmission coefficient $t=\sin \eta$ and reflection coefficient $r=\cos \eta$ through the input port $\mathbf{J}$ with light in the state $\left(\left|\frac{\alpha}{\cos \eta}\right\rangle_{J}-\left|-\frac{\alpha}{\cos \eta}\right\rangle_{J}\right)$, as shown in Fig [4

The unknown state of light wave $\mathbf{C}$ that Alice wants to teleport to Bob is $N_{\alpha x y}\left(x|\alpha\rangle_{C}+y|-\alpha\rangle_{C}\right) \equiv$ $N_{\alpha x y}\left(\begin{array}{l}x \\ y\end{array}\right)_{C}$, where $N_{\alpha x y}$ is the normalization constant $\left(N_{\alpha x y}=1 / \sqrt{|x|^{2}+|y|^{2}+\left(x^{*} y+x y^{*}\right) e^{-2|\alpha|^{2}}}\right)$. The total state of the light waves $\mathbf{A}, \mathbf{B}$ and $\mathbf{C},|\Psi\rangle_{A B C}=$ $\left|\Psi_{\eta}^{-}\right\rangle_{A B} N_{\alpha x y}\left(\begin{array}{l}x \\ y\end{array}\right)_{C}$, can be expanded as

$$
\begin{aligned}
|\Psi\rangle_{A B C}= & \frac{N_{\alpha \beta}^{-} N_{\alpha x y}}{2} \times \\
& \left\{\left(|\beta\rangle_{A}|-\alpha\rangle_{C}-|-\beta\rangle_{A}|\alpha\rangle_{C}\right)\left(\begin{array}{c}
x \\
y
\end{array}\right)_{B}\right. \\
& -\left(|\beta\rangle_{A}|-\alpha\rangle_{C}+|-\beta\rangle_{A}|\alpha\rangle_{C}\right)\left(\begin{array}{c}
x \\
-y
\end{array}\right)_{B} \\
& +\left(|\beta\rangle_{A}|\alpha\rangle_{C}-|-\beta\rangle_{A}|-\alpha\rangle_{C}\right)\left(\begin{array}{c}
y \\
x
\end{array}\right)_{B} \\
& \left.+\left(|\beta\rangle_{A}|\alpha\rangle_{C}+|-\beta\rangle_{A}|-\alpha\rangle_{C}\right)\left(\begin{array}{c}
-y \\
x
\end{array}\right)_{B}\right\}
\end{aligned}
$$

The four Bell-type states which need to be distinguished here are $\left(|\beta\rangle_{A}|-\alpha\rangle_{C} \mp|-\beta\rangle_{A}|\alpha\rangle_{C}\right)$ and $\left(|\beta\rangle_{A}|\alpha\rangle_{C} \mp|-\beta\rangle_{A}|-\alpha\rangle_{C}\right)$.

We recall that the four "quasi-Bell states" $\left(|\alpha\rangle_{A}|-\alpha\rangle_{C} \mp|-\alpha\rangle_{A}|\alpha\rangle_{C}\right) \quad$ and $\quad\left(|\alpha\rangle_{A}|\alpha\rangle_{C} \quad \mp\right.$ $|-\alpha\rangle_{A}|-\alpha\rangle_{C}$ ) can all be distinguished (with a high probability if $\alpha$ is not too small) by a linear optical scheme using a 50/50 beam splitter and two detectors $\left(\mathbf{B S}_{\mathbf{1}}, \mathbf{D}_{\mathbf{E}}\right.$ and $\mathbf{D}_{\mathbf{F}}$ of Fig [5) 11, 12], provided that the two detectors $\mathbf{D}_{\mathbf{E}}$ and $\mathbf{D}_{\mathbf{F}}$ can discriminate between odd and even numbers of photons. The corresponding generalized measurement to distinguish all four quasi-Bell-type states $(|\beta\rangle|-\alpha\rangle \mp|-\beta\rangle|\alpha\rangle)$ and $(|\beta\rangle|\alpha\rangle \mp|-\beta\rangle|-\alpha\rangle)$ can be accomplished by adding a second beam splitter of transmission coefficient $t=\tan \eta$ and an additional detector that can also discriminate between odd and even photons $\left(\mathbf{B S}_{\mathbf{2}}\right.$ and $\mathbf{D}_{\mathbf{Y}}$ of Fig $\mathbf{5}$ ) in the path of light wave $\mathrm{C}$, as shown in Fig 5 A straightforward calculation yields that the state $|\Psi\rangle_{A B C}$ of Eq. (8) is transformed, via the action of $\mathbf{B S}_{\mathbf{1}}$ and $\mathbf{B} \mathbf{S}_{\mathbf{2}}$, into $|\Psi\rangle_{E F Y B}$, where

$$
\begin{aligned}
|\Psi\rangle_{E F Y B}=\frac{N_{\alpha \beta}^{-} N_{\alpha x y}}{2} & \left\{|0\rangle_{E}\left(|-\sqrt{2} \beta\rangle_{F}|-r \alpha\rangle_{Y}-|\sqrt{2} \beta\rangle_{F}|r \alpha\rangle_{Y}\right)\left(\begin{array}{c}
x \\
y
\end{array}\right)_{B}\right. \\
& -|0\rangle_{E}\left(|-\sqrt{2} \beta\rangle_{F}|-r \alpha\rangle_{Y}+|\sqrt{2} \beta\rangle_{F}|r \alpha\rangle_{Y}\right)\left(\begin{array}{c}
x \\
-y
\end{array}\right)_{B} \\
& +|0\rangle_{F}\left(|\sqrt{2} \beta\rangle_{E}|r \alpha\rangle_{Y}-|-\sqrt{2} \beta\rangle_{E}|-r \alpha\rangle_{Y}\right)\left(\begin{array}{c}
y \\
x
\end{array}\right)_{B} \\
& \left.+|0\rangle_{F}\left(|\sqrt{2} \beta\rangle_{E}|r \alpha\rangle_{Y}+|-\sqrt{2} \beta\rangle_{E}|-r \alpha\rangle_{Y}\right)\left(\begin{array}{c}
-y \\
x
\end{array}\right)_{B}\right\}
\end{aligned}
$$

$\begin{array}{lrrr}\text { where } r & =\quad \sqrt{1-\tan ^{2} \eta} \text {. } & \text { Noting } & \text { that } \\ \left(|u\rangle_{F}|v\rangle_{Y} \mp|-u\rangle_{F}|-v\rangle_{Y}\right) & \text { can } & \text { be } & \text { writ- } \\ \text { ten as } \quad \frac{1}{2}\left[\left(|u\rangle_{F} \mp|-u\rangle_{F}\right)\left(|v\rangle_{Y}+|-v\rangle_{Y}\right)\right. & +\end{array}$
$\left.\left(|u\rangle_{F} \pm|-u\rangle_{F}\right)\left(|v\rangle_{Y}-|-v\rangle_{Y}\right)\right]$, all four cases represented by the four lines of Eq.(9) can be discriminated by observing which of the three detectors $\mathbf{D}_{\mathbf{E}}, \mathbf{D}_{\mathbf{F}}$ and 


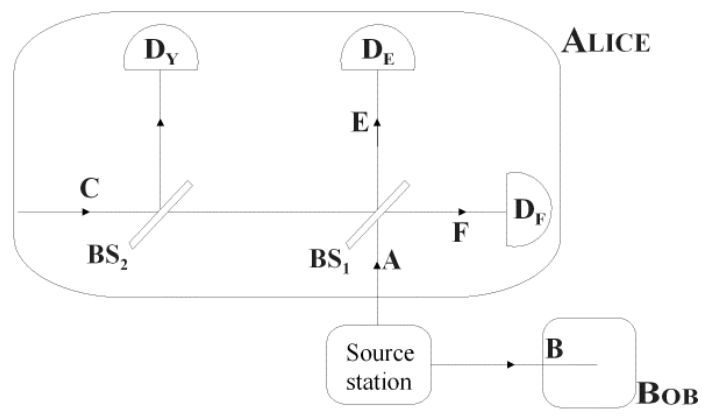

FIG. 5: Linear optical scheme for a conclusive teleportation of the state $\left(x|\alpha\rangle_{C}+y|-\alpha\rangle_{C}\right)$. The source station generates a nonmaximally entangled state $\left|\Psi_{\eta}^{-}\right\rangle_{A B}=$ $N_{\alpha \beta}^{-}\left(|\beta\rangle_{A}|-\alpha\rangle_{B}-|-\beta\rangle_{A}|\alpha\rangle_{B}\right)$, where $\beta=\alpha \tan \eta$. $\quad \mathbf{D}_{\mathbf{E}}$, $\mathbf{D}_{\mathbf{F}}$, and $\mathbf{D}_{\mathbf{Y}}$ are detectors, $\mathbf{B S}_{\mathbf{1}}$ is a $50 / 50$ beam splitter, and $\mathbf{B S}_{2}$ is a beam splitter of transmission coefficient $t=\tan \eta$.

TABLE I: Possible outcomes of the detectors $\mathbf{D}_{\mathbf{E}}, \mathbf{D}_{\mathbf{F}}$ and $\mathbf{D}_{\mathbf{Y}}$ and corresponding states of light wave $\mathbf{B}$

\begin{tabular}{cccc}
\hline \hline $\mathbf{D}_{\mathbf{E}}$ & $\mathbf{D}_{\mathbf{F}}$ & $\mathbf{D}_{\mathbf{Y}}$ & State of $\mathbf{B}$ \\
\hline \hline 0 & odd & even & $x|\alpha\rangle_{B}+y|-\alpha\rangle_{B}$ \\
0 & even & odd & \\
\hline 0 & even & even & $x|\alpha\rangle_{B}-y|-\alpha\rangle_{B}$ \\
0 & odd & odd & \\
\hline odd & 0 & even & $x|-\alpha\rangle_{B}+y|\alpha\rangle_{B}$ \\
even & 0 & odd & \\
\hline even & 0 & even & $x|-\alpha\rangle_{B}+y|\alpha\rangle_{B}$ \\
odd & 0 & odd & \\
\hline \hline
\end{tabular}

$\mathbf{D}_{\mathbf{Y}}$ registers zero, odd or even number of photons. The possible measurement outcomes are summarized in Table 【 The only case in which the generalized measurement fails is when the two detectors $\mathbf{D}_{\mathbf{E}}$ and $\mathbf{D}_{\mathbf{F}}$ both register zero photon. The success probability $P$ is thus given by $\left(P=1-\right.$ Probability for both $\mathbf{D}_{\mathbf{E}}$ and $\mathbf{D}_{\mathbf{F}}$ to detect no photon). While $P=\sin ^{2} \eta$ for the previous two cases (a) and (b), the success probability for the present case will show a different behavior with respect to $\eta$. Details of the comparison will be given in the next section.

\section{DISCUSSION}

We have presented linear optical schemes for conclusive teleportation when Alice and Bob share nonmaximal entanglement. Considered in detail are three different cases when two basis states for a qubit are (a) $|1\rangle$ and $|0\rangle$, (b) $|V\rangle$ and $|H\rangle$, and (c) $|\alpha\rangle$ and $|-\alpha\rangle$. The essence of the schemes common to all three cases is the use of a beam splitter of an appropriate value of transmission coefficient to reduce the amplitude of one part of the entangled state, consistent with the idea of loss-induced generalized measurement $[8$. There exist, however, some interesting differences in details of the schemes between

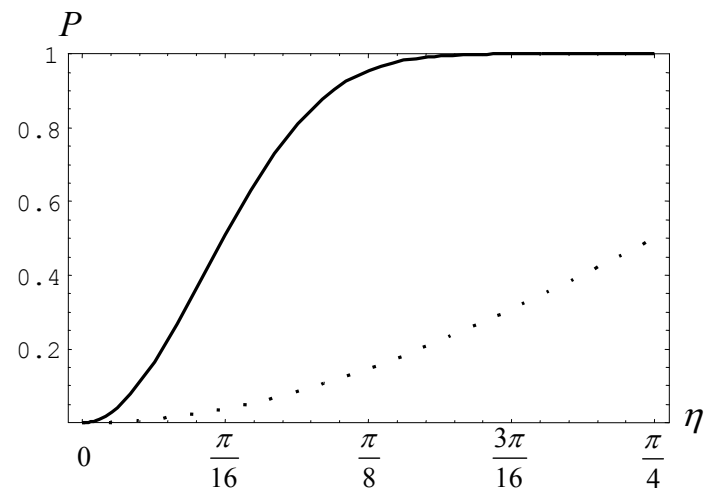

FIG. 6: The success probability $P$ vs. $\eta$ for case (c) with $\alpha=3$ (solid curve) and for cases (a) and (b) (dotted curve).

the three cases. We have found that, while for case (a) an additional detector that can distinguish between zero and one photon is needed in addition to the beam splitter, for case (b) no additional detector is required. The success probability in both cases (a) and (b) decreases as $\sin ^{2} \eta$ as the degree of entanglement is decreased. In contrast to this, the success probability for case (c) behaves in a more complicated way, as the generalized measurement in this case fails only when the two detectors $\mathbf{D}_{\mathbf{E}}$ and $\mathbf{D}_{\mathbf{F}}$ both register zero photon. It is interesting to note that the failure of the standard measurement to distinguish the four quasi-Bell states, $(|\alpha\rangle|-\alpha\rangle \mp|-\alpha\rangle|\alpha\rangle)$ and $(|\alpha\rangle|\alpha\rangle \mp|-\alpha\rangle|-\alpha\rangle)$, in case of maximal entanglement is also subject to the same condition, i.e., the two detectors $\mathbf{D}_{\mathbf{E}}$ and $\mathbf{D}_{\mathbf{F}}$ registering no photon 11,12$]$. Nevertheless, the success probability decreases as the degree of entanglement decreases, because the probability for the two detectors $\mathbf{D}_{\mathbf{E}}$ and $\mathbf{D}_{\mathbf{F}}$ to detect no photon increases as the parameter $\beta(=\alpha \tan \eta)$ decreases. We show in Fig 6 the success probability as a function of $\eta$ for case (c) where $\alpha=3$ is assumed, along with the curve $\sin ^{2} \eta$ representing the success probability for cases (a) and (b). One first notes that the success probability for case (c) is nearly 1 when $\eta=\frac{\pi}{4}$, whereas that for cases (a) and (b) is at most $1 / 2$, as long as only linear optical schemes are employed. Furthermore, the success probability for case (c) decreases much more slowly than that for cases (a) and (b), as $\eta$ is decreased from $\frac{\pi}{4}$. In other words, the success probability for case (c) remains much closer to its maximum value for the same amount of amplitude errors. This robustness of the success probability with respect to amplitude errors stems largely from the robustness of the degree of entanglement with respect to amplitude errors. In fig $[7$ we show the degree of entanglement, $E=-\operatorname{Tr}(\rho \log \rho)$, with respect to $\eta$ for the nonmaximally entangled states (7) and (1). The degree of entanglement remains close to 1 for a relatively broad range of $\eta$ for state (7), while it decrease rapidly as $\eta$ is decreased from $\frac{\pi}{4}$ for state (11). Fig 8 shows the success probability as a function of the degree of entanglement for case (c) and for cases (a) and (b). Regardless of the 


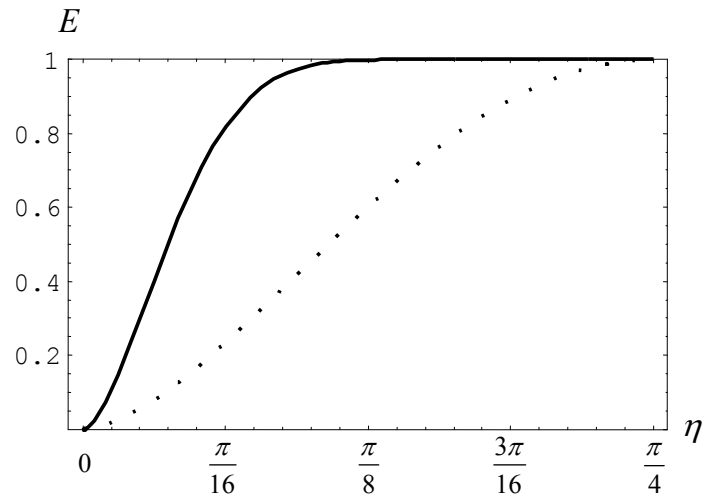

FIG. 7: The degree of entanglement $E$ vs. $\eta$ for state (7) (solid curve) and for state (1) (dotted curve).

value of the degree of entanglement, the success probability for case (c) is roughly twice that for cases (a) and (b). We mention that the behavior of the success probability for case (c) exhibited in Figs 6 and 8 remains essentially the same, as long as $\alpha \gtrsim 3$. We also mention that the success probability for case (c) varies little with respect to the unknown amplitudes $x$ and $y$, as long as $\alpha \gtrsim 3$.

It should be noted that our observation that the success probability for case (c) remains close to 1 over a relatively broad range of the amount of amplitude error stands only if we have photon detectors which can distinguish between even and odd numbers of photons. At present, photon detectors with single photon resolution which can distinguish between $n$ and $(n+1)$ photons do not exist, despite recent efforts and progress 15, 16]. It is certainly a high technical challenge to construct such detectors. It is, however, in principle possible to distinguish between $n$ and $(n+1)$ photons, if one uses photon counters that have been developed to distinguish between no photon and a single photon or between a single photon and two photons with high quantum efficiency 15] in an arrangement of detector cascades or $N$-ports [17]. It has also been suggested that $n$ and $(n+1)$ photons can be distinguished by utilizing homodyne detection looking at the imaginary quadrature 18] or by coupling the field to a two-level atom through nonlinear interaction [12, 19].

In conclusion, we have presented experimental schemes to perform generalized measurements for conclusive teleportation when the sender and the receiver share nonmaximally entangled states for three different cases. Our analysis shows that the scheme with a coherent state qubit is relatively robust against amplitude errors. This advantage may play an important role in actual implementation of linear optical schemes for quantum communication and quantum computation 18, 20, 21 based on coherent-state ouhits

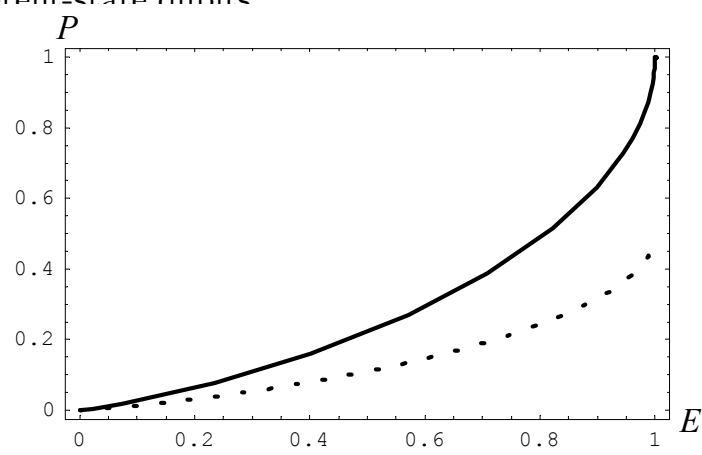

FIG. 8: The success probability $P$ vs. the degree of entanglement $E$ for case (c) (solid curve) and for cases (a) and (b) (dotted curve)

\section{Acknowledgments}

This research was supported by Korea Research Foundation under Contract No. 2002-070-C00029.
[1] C.H. Bennett, G. Brassard, C. Crepeau, R. Jozsa, A. Peres, and W.K. Wootters, Phys. Rev. Lett. 70, 1895 (1993).

[2] D. Bouwmeester , J.W. Pan, K. Mattle , M. Eibl ,H. Weinfurter , and A. Zeilinger, Nature 390, 575 (1997); D. Boschi, S. Branca, F. De Martini, L. Hardy, and S. Popescu, Phys. Rev. Lett. 80, 1121 (1998).

[3] E. Lombardi, F. Sciarrino, S. Popescu, and F. De Martini, Phys. Rev. Lett. 88, 070402 (2002).

[4] A. Furusawa, J.L. Sorensen, S.L. Braunstein, C.A. Fuchs, H.J. Kimble, and E.S. Polzik, Science 282, 707 (1998).

[5] T. Mor and P. Horodecki, quant-ph/9906039.

[6] C.H. Bennett, H.J. Bernstein, S. Popescu, and B. Schumacher, Phys. Rev. A 53, 2046 (1996).

[7] W.L. Li, C.F. Li, and G.C. Guo, Phys. Rev. A 61, 034301 (2000).

[8] B. Huttner, A. Muller, J.D. Gautier, H. Zbinden, and N. Gisin, Phys. Rev. A 54, 3783 (1996).

[9] H.W. Lee and J. Kim, Phys. Rev. A 63, 012305 (2000).
[10] K. Mattle, H. Weinfurter, P.G. Kwiat, and A. Zeilinger, Phys. Rev. Lett. 76, 4656 (1996).

[11] S.J. van Enk and O. Hirota, Phys. Rev. A 64, 022313 (2001); O. Hirota, S.J. van Enk , K. Nakamura , M. Shoma , and K. Kato, quant-ph/0101096 H. Jeong, M.S. Kim, and J. Lee, Phys. Rev. A 64, 052308 (2001); N.B. An, Phys. Rev. A 68, 022321 (2003); Y.W. Cheong, H. Kim, and H.W. Lee, in preparation.

[12] X. Wang, Phys. Rev. A 64, 022302 (2001)

[13] A.G. White, D.F.V. James, P.H. Eberhard, and P.G. Kwiat, Phys. Rev. Lett. 83, 3103 (1999).

[14] S. Glancy, H. Vasconcelos, and T.C. Ralph, quant-ph/0311093

[15] P.G. Kwiat, A.M. Steinberg, R.Y. Chiao, P.H. Eberhard, and M.D. Petroff, Phys. Rev. A 48, R867 (1993); S. Takeuch, J. Kim, Y. Yamamoto, and H.H.Hogue, Appl. Phys. Lett. 74, 1063 (1999); J. Kim, S. Takeuch, Y. Yamamoto, and H.H. Hogue, Appl. Phys. Lett. 74, 902 (1999). 
[16] A.J. Miller, S.W. Nam, J.M. Martinis, and A.V. Sergienko, Appl. Phys. Lett. 83, 791 (2003); J. Rehacek, Z. Hradil, O. Haderka, J. Perina Jr., and M. Hamar, quant-ph/0303032 H. Lee, U.H. Yurtsever, P. Kok, G.M. Hockney, C. Adami, S.L. Braunstein, and J.P. Dowling, quant-ph/0310161 D. Achilles, C. Silberhorn, C. Sliwa, K. Banazek, I.A. Walmsley, M.J. Fitch, B.C. Jacobs, T.B. Pittman, and J.D. Franson, quant-ph/0310183

[17] S. Song, C.M. Caves, and B. Yurke, Phys. Rev. A 41, R5261 (1990); M. Reck, A. Zeilinger, H.J. Bernstein, and P.Bertani, Phys. Rev. Lett. 73, 58 (1994); H. Paul,
P. Torma, T. Kiss, and I. Jex, Phys. Rev. Lett. 76, 2464 (1996); P. Kok and S.L. Braunstein, Phys. Rev. A 63, 033812 (2001).

[18] T.C. Ralph, A. Gilchrist, G.J. Milburn, W.J. Munro, and S. Glancy, quant-ph/0306004

[19] B. Yurke and D. Stoler, Phys. Rev. A 35, 4846 (1987); B. Yurke and D. Stoler, Phys. Rev. Lett. 79, 4941 (1997); C. C. Gerry, Phys. Rev. A 61, 043811 (2000).

[20] H. Jeong and M.S. Kim, quant-ph/0109077

[21] E. Knill, R. Laflamme,and G.J. Milburn, Nature, 409, 46 (2001). 